bution, a more local neurosis would accord better with the clinical facts; but even now we have only gone one step further back, for while the nervous system may start a morbid process, and may have influence in localising its cutaneous manifestations, some other factor is necessary to determine the kind of disease that shall ensue. What that other factor is we do not know ; probably the idiosyncrasy of the patient, whether congenital or acquired, is one of the most potent influences. In this case our French confrères, from its relation to rheumatism, would probably consider that the arthritic disposition was the factor sought for ; but, as I have already shown, this is not a satisfactory explanation, for it has already been made responsible for so many rarieties of skin eruption that it leaves us much in the same position as before.

There is another point in these cases to which I must allude. Of the four cases before you, three are females and one a male. As it happens, this is the proportion in which, vaking a large number of cases, the disease affects the different sexes, and the coincidence will perhaps impress it upon your minds.

Such then, gentlemen, are the history and explanation, as far as I can afford it, of this mysterious disease, which, attacking people in the prime of life who were often previously in good health, too frequently leaves them hopeless cripples and blights their whole future existence. Is there, then, nothing to be done for these unfortunate patients? Yes, something may, though it falls far short of what we should wish. For the hypertrophic cases there is always hope that improvement may occur before new fibrous tissue is completely formed and undergoes its subsequent contraction. The first two cases show that nature itself does much but we may assist her, not so much directly as indirectly. Good food and protection from changes of temperature are imperative; ferruginous and other tonics, with cod-liver oil, are distinctly beneficial in most cases, and shampooing after Turkish baths, or friction with oily substances appear to be important adjuncts; while some attach importance to galvanisation of the sympathetic. Although it is difficult to appraise the value of these measures in a disease capable of spontaneous improvement, yet as a whole I believe them to be useful, and they are certainly grateful and encouraging to the patient to whom a "do-nothing" treatment is profoundly disheartening. For the atrophic cases the outlook is decidedly gloomy, too often by this time irremediable crippling has resulted before the patient is seen. Priscilla $\mathrm{F}$ s case should encourage us to strive to give relief, though a cure is hopeless, for she had had the disease three years without improvement, when she first came under observation, and now has reoained many of the lost movements, and all induration has disappeared. The same line of treatment was pursued as that indicated above, except that she had only the frictions and tonics without either Turkish baths or galvanism. The last agent is much adrocated by some observers, but I have not tried it in these cases; for Jane $\mathrm{R}$ - was so evidently improving spontaneously that it would have only been misleading to have used such an agent, while Jane E- had been treated by galvanism for twelve months before she came here, without benefit. The best proof we can get of the efficacy of any line of treatment is when, after the disease has been quite stationary for some time, improvement ensues on the adoption of a certain measure. This is the case already with Jane E-_; so that I think we may fairly credit the means employed with a good share in her improvement. The use of Turkish baths can afford another test of improvement-namely, in the resumption of the secretion of sweat, which is in abeyance when the disease exists in a high degree. Jane $\mathbf{R}-$, for instance, observed spontaneously that sweating had returned in her hands in one of the baths, after being absent for several months. I think, therefore, there is fair ground for assuming that these therapeutic measures do really influence the disease for good, probably by improving the impeded circulation. of course, arsenic, iodide of potassium, and mercury have been administered over and over again, but there is no evidence that they have been of any benefit; and mercury appears to have been distinctly injurious.

AT the recent annual meeting of the supporters of the Windsor Dispensary and Infirmary a very farourable report was presented, showing a balance in hand. During the past year a new women's ward has been erected, and increased facilities for dealing with accident cases provided. \section{进ettsomian 迹ectures}

ABSTRACT OF

on

\section{DISORDERS OF DIGESTION,} THEIR CONSEQUENCES AND TREATMENT.

\section{Delivered at the Medical Society of London on Monday, February $2 n d, 1885$,}

By T. LAUDER BRUNTON, M.D., F.R.C.P., ASSISTANT-PHYSICIAN TO ST. BARTHOLOMEW'S HOSPITAL.

\section{LECTURE III.}

WITH regard to the treatment of disturbances in the digestive function, the first step towards restoring it to health is to remove, if possible, the disturbing causes. Imperfect mastication is one of the commonest causes of disordered digestion. Man is a low-pressure engine, and works almost all his organs considerably under their full power. A healthy stomach can usually digest a good deal more than it is commonly called upon to do. There is, however, a limit to all things, and that limit is more easily reached in some cases than in others. The reserve powers of any function gradually diminish as age increases. If the mind be excited in any way, the movements of mastication are apt to sympathise with the mental excitement. It is evident that, during meals, all ideas of action to be taken by the individual himself should be banished from the mind. Reading anything which interests strongly should not be done during meal-time; and solitary meals are as a rule to be avoided, for the presence of a companion serves to maintain the nervous activity to which reference was made in the first lecture as an important factor in perfect digestion. The condition of the teeth is another cause of imperfect mastication. No teeth at all is far better than a few teeth; an absolutely toothless old man may masticate and enjoy eren hard toast, and here the gums may be like the mandibles of a turtle. The nature of the food plays a considerable part in the process of perfect mastication; for if the meat be tough or the bread new proper comminution is impossible, and so digestion is more difficult. The fine subdivision of fatty food is also of great importance in regard to its digestion. The fat of hot mutton is indigestible because it cannot be pulverised, whilst cold fat can. The more minutely we can subdivide the fat the more easily it is digested. Many years ago Professor Hugo Kronecker asked Dr. Brunton: "How should butter be spread in a sandwich? Should the whole of it be put on one slice of bread and the other slice of bread simply put over the top of it, or should the pat of butter be divided into halves and one of them spread on each piece of bread?" The professor answered the question: "The butter should be divided into halres, and one spread on each piece of bread, because in this way the butter is more minutely subdivided, and thus not only gives a more agreeable taste, but is more readily digested." In regard to butchers' meat there are great differences, depending both on the kind of meat used and its condition at the time of cooking. Meat cooked in a state of rigor mortis was tough to eat, but the old Romans used to eat still newer meat; they suffocated their fowls in wine and cooked them forthwith, so that both meat which is perfectly freshly killed, and also meat which has been kept for a sufficient time, are tender. Dr. Brunton thought the taste for "high" meat was an acquired and morbid one. Eating high meat may overtax the antiseptic powers of the gastric juice and bile. The Esquimaux and Icelanders can consume decomposing food to an extraordinary extent without suffering. The effect of keeping may, to a certain extent, be imitated by the application of a vegetable digestive ferment. In the West Indies a tough beefsteak is rendered tender by rubbing it with the juice of a fresh papaw fruit, which contains a ferment, papaine, having an action very much like the trypsin of the pancreas. Bad cooking is another cause of imperfect digestion. Pleasant and repulsive food will act on the stomach through the nerre centres; while the idea of pleasant food will excite 
appetite, the very idea of unpleasant food will excite disgust, and even bring on nausea and vomiting. The appearance of the table furniture may also exert an influence on the digestion. That food itself is a moral agent has long been recognised, and has found expression in the proverb, "A hungry man is an angry man." The favour with which Isaac blessed Jacob was augmented by the ingestion of savoury meats. Cookery may not only be a powerful moral agent in regard to individuals, but may be of great service in regenerating a nation. "Schools of cookery for the wives of working men in this country will do more to abolish drinking habits than any number of teetotal associations." A clear-headed clergyman in New York has perceived that dentistry may be a moral agent, and he has insisted on all the people attending his mission chapel keeping their teeth in good condition. ${ }^{1}$ Since the clergyman adopted this plan he has had very much less trouble from drunkenness in his congregation. In Switzerland the relation between the consumption of alcohol and the quality of the cookery has recently been investigated. Savoury substances probably stimulate the circulation of the blood in the nervous centres.

Another important question is the kind of food which a person may eat. The appetite is the best guide for the healthy man. As Dr. Austin Flint very sensibly puts it, "the diet should be regulated by the appetite, the palate, and by common sense." The duration of the meal is an important item, and the comfort of digestion at a table d'hote may be safely attributed to the forced slowness of the progress of the meal.

The pleasurable sensation of perfect satisfaction, ease, and quiescence of body and mind should be the criterion of sufficient alimentation. To effect this most agreeable of sensations and conditions- the real elysian satisfaction of the reasonable epicure - timely attention must be paid to the preliminary processes, such as thorough mastication and moderate or slow deglutition.

Many a man has been saved much misery by a weak stomach, which punished its owner by sickness or headache whenever he tried to orerburden it, and thus checked his tendency towards excess at the very outset. The late Professor Laycock observed that patients recovering from a severe illness not infrequently have a craving for salt herrings, pork, or ham; but the fact is that these patients really want salt, and if salt be given them in the form of a mixture the appetite is appeased and the harm is avoided which the herring or ham might have caused. The degrees of digestibility of various meats, fruits, vegetables, and drinks were discussed at length. Sour wines are apt to set up gastric catarrh. A single glass of good wine sometimes disagrees, and some of the people who suffer in this manner also have an unpleasant burning feeling in the gullet whilst swallowing the wine, and Dr. Brunton suggests that hyperæesthesia of the mucous membrane may have something to say in the matter. Coffee does not affect the stomach to such an extent as tea. In its preparation, however, a substance called caffeon is produced, and this, along with the caffein which is present in coffee and tea, appears to dilate the abdominal vessels and cause a feeling of fulness in the abdomen, with a tendency to piles in some persons.

Another cause of imperfect indigestion is fatigue. When exhausted, it is far better to get forty winks before dinner than after. Walking or other exercise after a long day's work and prior to dinner, by exhausting the nervous system, was a potent cause of indigestion. Effects somewhat similar to those of fatigue may be produced by depressing or disturbing mental emotions or bodily conditions. Different emotions appear to affect specially, not only different organs like the heart and intestinal canal, but different parts of the digestive apparatus. Thus disgust affects the stomach, causing vomiting; fear is seen, in some of the lower animals, to affect the rectum, causing defecation; compassion affects the small intestine, producing borborygmus; worry and anxiety appear to have a very special influence upon the liver. In treating indigestion due to injurious mental influences, bromide of potassium, either alone or combined with bromide of ammonium, is very useful, both in lessening the sensibility of the nervous system to worry and in procuring sleep; for, as Shakespeare truly says,

Sorrow's weight doth heavier grow

Through debt that bankrupt sleep doth sorrow owe."

Disorders of the genital organs are apt to give rise to mental depression and to digestive derangements.
The pathology of indigestion and the action of remedies cannot be properly understood unless we constantly bear in mind the intimate relation which exists between the alimentary canal and the rest of the body.

The gastric tonics are chiefly vegetable bitters, and they increase the appetite, lessen flatulence, and tend to diminish discomfort and languor. Nux vomica is one of the most useful of this class, and the great benefit derived from its use is probably due to its stimulating action on the nerve centres, by which the coördination of the digestive processes is rendered more perfect. Carminatives tend to disperse flatulence; the ethers and volatile oils probably act by increasing the movements of the stomach and intestine, and altering them in such a way as to allow the gases they contain to escape upwards or downwards. Charcoal, subnitrate of bismuth, and binoxide of manganese would appear to act largely mechanically; and cases of dyspepsia are reported which have been successfully treated by the administration of fine sand. The ethers and alcohols are closely allied to the carminatives. The difficulty about alcohol is to define its place. There is a great deal of practical truth in the definition of dirt as " matter in the wrong place." In regard to the use of alcohol in dyspepsia, Dr. Brunton thought St. Paul's advice to Timothy was very good: "Drink no longer water, but use a little wine for thy stomach's sake and thine often infirmities." Another important chapter in treatment is the removal of waste products. The regular action of the bowels must be maintained at all cost. Headache from constipation is probably due, in part at least, to poisonous products formed in the intestine and absorbed from it, for Brieger noticed that it was only in the first stages of albuminous decomposition that alkaloids are formed, and afterwards they seemed to disappear. So that prolonged constipation without headache may be explained. The manner in which the salts of Carlsbad are used at the site of their natural origin accounts for the greater benefit derived from them thar when they are used at home. The sipping has a powerful influence on the heart, as already pointed out in the first lecture.

One cause of biliousness, which deserves attention, is the alteration in the condition of the bile itself. Biliousness is accompanied by two different conditions of biliary flow. In the one kind the stools are clay-coloured from the absence of bile; in the other, the stools are either normal or are dark-coloured, from excess of bile. Certain bodies of the aromatic series have a powerful action upon the secretion of bile. Salicylate of soda is a powerful hepatic stimulant, increasing greatly the quantity and rendering very fluid the consistence of the bile. Other substances of the aromatic series, especially toluylendiamin, greatly increase the quantity of solids in the bile, so that sometimes the bile, from being viscid and thick, will no longer flow through the biliary capillaries, and jaundice results. A similar action may be exerted by poisons manufactured in the intestines. Mercurials may have an antiseptic action in addition to their other well-known effects. Horse exercise acts in a mechanical manner on the liver, tending to squeeze the bile out of the organ into the intestines; rowing, or its imitation in the gymnasium, may effect the same purpose. The alteratives, though ill-understood as is the manner of their action, must occupy an important place. The acids are probably a means of carrying out nitrogen in the form of ammonia, nitrogen which should normally pass out as urea. Nitro-hydrochloric acid is found to be clinically useful in patients suffering from eructations of sulphuretted hydrogen; it is also useful in oxaluria and depression of spirits. Ammonia and chloride of ammonium have a powerful action on the liver. Ammoniacal salts increase the formation of glycogen. Diuretics are useful in some cases of indigestion. Hot water is thus useful in some cases of gouty dyspepsia. .Alkalies increase the diuretic effect, and effervescent citrate or tartrate of potash is useful both as a diuretic and as a local sedative to the stomach in neurotic and gouty gastralgia. By the frequent use of water as a diluent, either alone or with salines, the consequences of indigestion in regard to the lungs, heart, and head may often be averted or remedied. Asthma in gouty subjects is, perhaps, best treated by a mixture of bromide and iodide of potassium, and the addition of a little arsenic is said to increase the effect. Bromide of potassium is frequently useful in cases of intermittent pulse, though Dr. W. Begbie used with success a remedy composed of two grains of powdered rhubarb, ten of subnitrate of bismuth, one and a half of nux vomica, and three of compound cinnamon powder, to be taken before meals, and, if there 
be acidity, ten grains of bicarbonate of soda or of magnesia may be added to it. It may be given in water or in a wafer swallowed along with a little water. Giddiness frequently takes the place of headache in persons of middle age suffering from biliousness, and both headache and giddiness are frequently connected with disorders of vision. Bilious headaches with tenderness of the globe of the eye may be relieved by small doses of salicylate of soda, half a grain in an ounce of water being taken every quarter of an hour or half an hour. Considerable attention ought also to be oiven in confirmed cases of indigestion to a most important method first introduced to notice by Weir Mitchell-namely, massage. A case of most profound emaciation, apparently dependent upon tubercular peritonitis, was narrated by Dr. Brunton in which the effects of massage seemed little short of marvellous. From the hasty sketch given of the disorders of digestion Dr. Brunton thought it would appear that although our knowledge of the subject is still very imperfect, yet a large number of observations have been accumulated which we may hope will, before long, enable us to understand the pathology more fully and to treat these disorders more perfectly.

$\mathrm{ON}$

\section{SOME COMPLICATIONS OF TRACHEOTOMY, WTTH ILLUSTRATIVE CASES.}

By ROBERT WILLIAM PARKER, M.R.C.S., SURGEON TO THE EAST LONDON HOSPITAL FOR CHILDREN.

$$
\text { (Concluded from p. 195.) }
$$

3. Irritative Changes due to the Presence of a Tube.-In a Iew cases the mere presence of a tracheotomy tube in the trachea, even when perfect as regards length, shape, and size, appears to give rise to an irritation which is quickly followed by the growth of granulations. A considerable amount of granulations has been found in from four to eight days in cases which have terminated fatally. Such a condition may be suspected if the insertion of the tube appears to cause undue irritation, and especially if its insertion is followed by blood-stained secretion. The use of the feather also causes very considerable irritation, and has to be discontinued. In cases where the granulations about the external wound proliferate with unusual exuberance, such a growth may be suspected within. This peculiarity seems to depend on a hypersensitiveness of the tracheal mucous membrane, and it is an idiosyncrasy which must be taken into account. Minor degrees of this condition are often experienced, some tracheotomised children never reconciling themselves to the cleaning and changing of the tube.

If granulations spring up, the best treatment is the local application of nitrate of silver. A silver probe, after being suitably bent and coated with fused nitrate of silver, is introduced into the tracheal wound, and made to touch as nearly as possible those parts on which the tube has rested. Or a few drops of a thirty-grain solution of nitrate of silver may be allowed to trickle into the trachea, the child sitting upright and inclined slightly backwards, so that the solution may run over the affected portion of the trachea.

The granulations I am now alluding to may occur all round the trachea, and not merely on the anterior wall or at one spot; for they are not due to pressure, but, as before stated, to the presence of a foreign body in an unusually sensitive trachea. I append the drawing of a trachea (see Fig. 5) in which granulations due to this cause were very abundant over the whole track of the tube. There was no sign of ulceration; the granulations occurred within five or six days of the operation. The child appeared to be doing very well, until one night it was suddenly choked after a violent fit of coughing. The autopsy revealed the presence of granulations between the tracheal wall and the tube, which doubtless during life were florid and turgid with blood, and completely filled the space; one currant-like granulation, larger than the rest and attached to a small pedicle, had apparently been driven by the coughing into the tube and completely occluded it.

In the case of warty laryngitis-that is, when there is a natural tendency to the formation of warts - the irritation of a tube leads to the production of minute warts, instead of granulations proper, as seen in Fig. 3, below the tracheal opening. In the case of granulations proper, some difficulty is usually met with in the removal of the tube. At first sight the obvious remedy is the removal of the cause; but an acute obstruction having necessitated the use of the tube, granulations having occurred and actually existing, the difficulty arises of how best to treat them. Such cases all point to the desideratum of tracheotomy without the use of tubes at all. At present, I have not been able to dispense with them; nor do I know of any trustworthy means of gaining the desired objects without tubes; at the same time it is a desideratum I constantly have in mind, and which I recommend to the consideration of my professional brethren.

As to treatment, reference has just been made to the use of nitrate of silver once a day, or once in two days. The alum spray or tannin spray (an ounce of tannic acid glycerine in seven ounces of water) must be used at frequent intervals through the day and night. The tube should be removed for as long as possible, and the silver plug inserted, the patient being meanwhile under direct supervision; for on the removal of the tube, the granulations swell out, and if present in quantity they in time occlude the trachea. Hence in a few instances a child will breathe well for an hour or two, then the tube must be reinserted. The explanation appears to be as follows. The tube by its presence keeps back the granulations, but having been removed, they commence to swell up; possibly also the more laboured breathing (which follows the removal of the tube), by causing congestion, helps them to still further occlude the

FIG. 5.

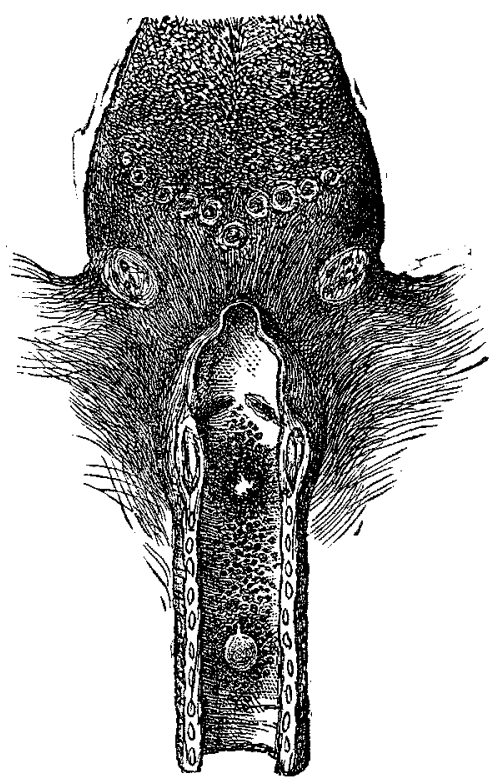

trachea. Cauterisation and astringents are the best remedies, and are generally successful.

4. Accidental Conditions. - Under this heading I shall include two cases, which, though not unique instances, are nevertheless so rarely met with as to require separate consideration. They appear so purely accidental in their incidence that none but the most general precautions can be taken to guard against them.

CAsw 6. Catarrhal Laryngitis; Tracheotomy ; Diffculty in removing Tube; Hypersensitiveness of the Trachea; Death in Convulsion fifteen days after removal of Tube; Autopsy; large Granuloma at the seat of cicatrised Wound inside the Trachea.-Eliza M-, aged two years and a half, was admitted into the hospital under the care of my colleague, Dr. Eustace Smith, on February, 1882. She was one of eight children, of whom one had recently been suffering from quinsy. She had enlarged glands about the neck, secondary to eczema capitis, to which disorder she was very subject. She was a plump, well-nourished child, and wellgrown for her age, with no very manifest signs of rickets. On admission she was said to have been hoarse for two days; she had that morning complained of having "a pin in her throat." She had had no discharge from her nose, and there was no illness in the house. Her breathing had become so laboured by the following day that tracheotomy had to be performed by Mr. Battams; the high operation was done; chloroform was administered by Mr. Sanderson, one of the resident officers. No membrane was got up or seen. The child did fairly well; efforts to remore the tube, however, 